administered intraperitoneally once chronic colitis had established (from day 35 post-infection, p.i.). Systemic, mesenteric lymph node (MLN) and colonic effects were analysed at day 45 p.i. MLN cell cytokine bead-array and colonic gene expression (RT-qPCR) analysis were performed. Colonic histopathology, tissue Foxp3 ${ }^{+}$and macrophage recruitment were determined. Treated groups were compared to naïve and untreated-infected $\operatorname{AKR}$ ( $n=5$ per group).

Results Neither treatment altered worm expulsion. Anti-TNF $\alpha \mathrm{Ab}$ and corticosteroid therapy preserved colonic length, compared to untreated disease. Colonic inflammation was less severe with steroid treatment $(p=0.005)$ and infliximab $(p=0.07)$. An increase in MLN TH2 cytokines was suggested with both treatments. Reduced colonic TNF $\alpha$, IL-1 $\beta$, IFN $\gamma$ and IL-12p40, and increased IL-13 expression were observed following Infliximab. Down-regulated TH1 cytokines, elevated TH2 cytokines (IL-4, IL-5, IL-13), and upregulated colonic IL-10 expression were detected following corticosteroid treatment. Colonic Foxp3 ${ }^{+}$cell numbers increased with disease but were unaltered by either treatment. A significant reduction in tissue $\mathrm{F} 4 / 80^{+}$macrophages was observed with infliximab treatment alone.

Conclusion Anti-TNF $\alpha \mathrm{Ab}$ and corticosteroid therapy suppress TH1-driven experimental colitis. Up-regulated transcription of TH2 and regulatory (IL-10, TGFb, Foxp3) pathway molecules was seen with corticosteroid treatment. This was not accompanied by an increased influx of Foxp3 ${ }^{+}$T-cell, suggestive that corticosteroids may alter regulatory-cell function more significantly than recruitment, in the reduction of pathology and disease activity. Anti$-\mathrm{TNF} \alpha \mathrm{Ab}$ treatment reduced colonic pro-inflammatory macrophage recruitment. With differing modalities of immunosuppression demonstrated, this model may increase understanding of why either mode of therapy can induce benefit in man even if the other has failed.

Competing interests None declared.

\section{PWE-246 INFLIXIMAB TREATMENT SIGNIFICANTLY REDUCES INFLAMMATORY MACROPHAGE NUMBERS WHILE PRESERVING REGULATORY MACROPHAGES IN A MOUSE MODEL OF CHRONIC CROHN'S COLITIS}

doi:10.1136/gutjnl-2012-302514d.246

${ }^{1} \mathrm{~S}$ Levison, ${ }^{2} \mathrm{M} \mathrm{C}$ Little, ${ }^{2} \mathrm{R} \mathrm{K}$ Grencis, ${ }^{1} \mathrm{~J} \mathrm{~T}$ McLaughlin, ${ }^{1} \mathrm{~J}$ L Pennock. ${ }^{1}$ Translational Medicine, University of Manchester, Manchester, UK; ${ }^{2}$ Manchester Immunology Group, University of Manchester, Manchester, UK

Introduction Inappropriate inflammatory responses to intestinal flora, augmented by host susceptibility genetics, contribute to the pathogenesis of Crohn's disease (CD). Transmural intestinal inflammation results from innate and adaptive immune cell infiltration, and pro-inflammatory cytokine accumulation. Activated macrophages represent a major source of $\mathrm{TNF} \alpha$ production. The treatment of CD with anti-TNF $\alpha$ antibody (Ab) therapy has proved clinically beneficial, yet over $30 \%$ of patients fail to respond. We characterised the biological and immunological effects of Infliximab therapy in a model of experimental colitis.

Methods Genetically identical mice (AKR), susceptible to chronic Trichuris muris-induced colitis, were infected with 300 T muris eggs. A single $5 \mathrm{mg} / \mathrm{kg}$ dose of Infliximab was administered intra-peritoneally once chronic colitis had established (from day 35 postinfection, p.i.). Systemic, mesenteric lymph node (MLN) and colonic effects were analysed at day 45 p.i. MLN cell cytokine bead-array and colonic gene expression (RT-qPCR) analysis were performed. Colonic histopathology, tissue Foxp ${ }^{+}$, and macrophage recruitment and phenotype were determined. The treatment group was compared to untreated-infected, naive, and naïve AKR administered Infliximab ( $\mathrm{n}=5$ per group).
Results Treatment did not alter worm expulsion. Anti-TNF $\alpha$ Ab therapy preserved colonic length compared to untreated disease $(p=0.049)$. Colonic inflammation was less severe with Infliximab treatment $(p=0.07)$. Reduced TNF $\alpha$, CCL2, and GMCSF proteins were measured in the MLN of Infliximab treated infected AKR. Reduced colonic expression of TNF $\alpha$, IL-1 $\beta$, IFN $\gamma$ and IL-12p40, and increased IL-13 was observed following Infliximab treated disease. Colonic Foxp3 ${ }^{+}$cell numbers increased with disease but were unaltered by treatment. Infected mice treated with Infliximab demonstrated a $50 \%$ reduction in colonic F $4 / 80^{+}$macrophages $(p=0.036)$. A relative increase of the proportion of colonic $\mathrm{Arg}^{+}$ alternatively activated macrophages (AAM $\Phi$ ) was observed with Infliximab treatment compared to untreated disease (29\% vs $14 \%$ ). Conclusion Infliximab therapy suppresses TH1-driven experimental colitis. Anti-TNFa Ab treatment reduced pro-inflammatory macrophages recruitment, and for the first time in vivo has been shown to preserve colonic tissue regulatory $A A M \Phi$. Whether a result of a fundamental alteration to macrophage recruitment, or the differentiation of a specific macrophage phenotype, requires further study. The presence of $A A M \Phi$ at index biopsy, or an increase in $A A M \Phi$ numbers following treatment initiation, may help to identify patient responders to Anti-TNF $\alpha$ Ab therapy.

Competing interests None declared.

\section{PWE-247 ARE WE MEASURING VITAMIN D IN INFLAMMATORY BOWEL DISEASE (IBD) PATIENTS?}

doi:10.1136/gutjnl-2012-302514d.247

S K Butt,* K Besherdas. Department of Gastroenterology, Chase Farm Hospital, London, UK

Introduction There is increasing interest in the role of vitamin D in IBD, outside of its traditional role in metabolic bone disease. Novel insights into additional roles for vitamin $\mathrm{D}$ are being established and these include anti-inflammatory and immune-modulating effects. Active vitamin $\mathrm{D}$ is known to exert its biological functions via the vitamin $\mathrm{D}$ receptor (VDR). Immune cells have been found to express VDR and possess the enzymes necessary to produce active vitamin D. This suggests vitamin D may have actions beyond endocrine activity. Furthermore, Vitamin D deficiency has been linked to higher rates of cancers including colorectal cancer. Previous studies have found that almost $50 \%$ of the IBD patients were vitamin $\mathrm{D}$ deficient at some point and 11\% were severely deficient. Vitamin D deficiency has been demonstrated to be independently associated with higher disease activity scores in patients compared to those that had normal levels of vitamin D. Furthermore, vitamin D deficient Crohn's patients have a poorer quality of life when compared to patients who are not vitamin D deficient. Currently, ECCO guidelines do not mention measurement of vitamin $\mathrm{D}$ in patients with IBD but given its effects, we set out to identify whether we were checking for and correcting for vitamin D deficiency in our IBD patients.

Methods The aim of the study was to investigate whether we were measuring vitamin $\mathrm{D}$ levels at any encounter in out IBD patients. This study was conducted in a busy District General Hospital in North London. Information was gathered using the hospital powerchart system and the IBD database of patients.

Results A total of 225 patients were correctly identified as having IBD. Of these, $157(70 \%)$ had Ulcerative colitis and $68(30 \%)$ had Crohn's disease. 24 (15\%) Ulcerative colitis patients and $8(12 \%)$ Crohn's patients had their vitamin D checked on hospital records. The range of vitamin $\mathrm{D}$ levels were 14-84 with lower limit of normal being 50. 13/32 (41\%) patients has low vitamin D levels Of these only two patients were also under the Rheumatology team for co-existing arthropathy/arthritis. 
Conclusion This study demonstrates that despite evidence of Vitamin D and its role in anti-inflammatory and immune-modulating effects (in addition to bone protection) we are not actively measuring and treating its deficiency. Perhaps guidance from ECCO may encourage our testing of vitamin D in IBD patients.

Competing interests None declared.

\section{PWE-248 DOCOSAHEXANOEIC ACID IN THE AETIOLOGY OF CROHN'S DISEASE-DATA FROM A EUROPEAN PROSPECTIVE COHORT STUDY (EPIC)}

doi:10.1136/gutjnl-2012-302514d.248

S Chan.* Department of Gastroenterology, Norfolk and Norwich University Hospitals NHS Foundation Trust, Norwich, UK

Introduction Docosahexaenoic acid (DHA) is an n-3 polyunsaturated fatty acid, present in fish oils, which possess anti-inflammatory properties, including biological effects on genetic expression, immune cell function and the production of inflammatory eicosonoids. The aim of this investigation was to conduct the first prospective cohort study to determine if low dietary intakes of this nutrient were associated with the development of incident Crohn's disease.

Methods A total of 229702 healthy participants aged 30-74 years of age were recruited in a prospective cohort study (EPIC-European Prospective Investigation Into Cancer) who were resident in either: Sweden, Denmark, The Netherlands, Germany or The UK. At baseline, participants completed food frequency questionnaires from which their intakes of DHA and other dietary fatty acids were determined. The cohort was followed-up between 1992 and 2004 to identify those who developed incident Crohn's disease, with each diagnosis medically confirmed by a review of the medical notes. Each case was matched with four controls for gender, age at recruitment and centre, and the analysis performed using conditional logistic regression. Adjustments were made for: smoking, total energy, fat and fatty acids, which affect the inflammatory process.

Results In the cohort, 73 participants developed incident Crohn's disease (64\% women) at a mean age of 56.3 years ( $\mathrm{SD}=11.1$ years). Of these, $51 \%$ had disease affecting the terminal ileum and $19 \%$ had a pancolitis. The four higher quintiles of DHA intake were all inversely associated with the development of Crohn's disease, with the highest (>310 mg/day) having the largest effect (OR 0.07, 95\% CI 0.01 to $0.80, p=0.03$ ), with a dose-response across categories (OR trend $0.54,95 \% \mathrm{CI} 0.30$ to $0.98, \mathrm{p}=0.04)$. The attributable fraction, namely the percentage of cases which may be due to the lowest dietary intake of DHA was $40 \%$.

Conclusion The data suggest a potential dose-dependent protective effect for increasing dietary DHA in the aetiology of Crohn's disease. DHA should be measured in future aetiological studies of this disease and could be assessed as a dietary treatment in clinical trials of patients.

Competing interests None declared.

\section{PWE-249 BODY MASS INDEX IN THE AETIOLOGY OF INFLAMMATORY BOWEL DISEASE-DATA FROM A EUROPEAN COHORT STUDY (EPIC)}

doi:10.1136/gutjnl-2012-302514d.249

S Chan.* Department of Gastroenterology, Norfolk and Norwich University Hospitals NHS Foundation Trust, Norwich, UK

Introduction There are plausible biological mechanisms for how obesity may be involved in the development of inflammatory bowel disease (IBD) due to the pro-inflammatory cytokines synthesised by adipose tissue including TNF- $\alpha$. The aim of this work was to conduct the first prospective cohort study of obesity in the aetiology of IBD.

Methods The cohort consisted of 366351 healthy men and women aged 30-74 years from centres in Europe (EPIC-European Prospective Investigation into Cancer) recruited between the years 1991 and 1998. At recruitment participants' weight, height and energy intake were measured and physical activity from questionnaires and their body mass index (BMI) calculated. The cohort was monitored to 2004 to identify those who developed Crohn's disease (CD) or ulcerative colitis (UC). Each diagnosis was medically confirmed via review of the medical notes. Each case was matched with four controls, for gender, age at recruitment and centre. BMI was divided into five categories. Conditional logistic regression was used to calculate OR and 95\% CI adjusted for smoking.

Results In the cohort, 102 participants developed incident CD (73\% female, mean age of 55.9 years, $\mathrm{SD}=11.0$ years) and 213 participants developed incident UC (61\% female, mean age of 57.5 years, $\mathrm{SD}=10.8$ years). There were no associations between the four higher categories of BMI and the development of CD (Trend $=1.00,95 \% \mathrm{CI}$ 0.79 to $1.27, p=0.91$ ) or UC (Trend $=1.03,95 \%$ CI 0.83 to $1.29, p$ 0.76). Dividing BMI in two categories.

Conclusion No associations were found between BMI and either CD or UC. Further studies are required to confirm these results. If confirmed then BMI does not need to be measured in future epidemiological studies of IBD.

Competing interests None declared.

\section{PWE-250 AZATHIOPRINE AND ALLOPURINOL CO-THERAPY FOR IBD PATIENTS IS A SAFE AND EFFECTIVE TREATMENT OPTION IN THE DISTRICT GENERAL HOSPITAL SETTING}

doi:10.1136/gutjnl-2012-302514d.250

H E Johnson, S A Weaver, S D McLaughlin. " Department of Gastroenterology, Royal Bournemouth Hospital, Bournemouth, UK

Introduction Reports from specialist inflammatory bowel disease (IBD) units have demonstrated that allopurinol and low-dose thiopurine co-therapy is an effective treatment option in patients who have failed standard dose therapy. There is little experience however from the district general hospital (DGH) setting. Co-therapy was introduced in our unit in September 2010.

Aim To evaluate the safety and therapeutic outcome of IBD patients treated with azathioprine and allopurinol co-therapy at our institution.

Methods A prospective database of all patients treated with allopurinol co-therapy is maintained at our institution. We reviewed the database entries and case notes of all patients. Data from patients who had been on allopurinol for $<3$ months were disregarded.

Results 25 patients were identified, five were excluded because of insufficient follow-up ( $<3$ months) and one patient was lost to follow-up. The median length of co-therapy was 9 (range 3-12) months. Diagnosis was ulcerative colitis (12), Crohn's disease (7), IBD-Unclassified (1). Indications for co-therapy were abnormal LFTs (3), drug side effects (8), high methylmercaptopurine (5), gout (2), therapeutic failure (2). 6-thioguanine nucleotide levels were measured where appropriate in patients before co-therapy, of these $50.0 \%$ were therapeutic. Following co-therapy 6-thioguanine nucleotide levels were therapeutic in $81.8 \%$ of patients. Co-therapy was effective and well tolerated in $13(68.4 \%)$. Two (10\%) patients developed side effects from allopurinol both had been treated with $200 \mathrm{mg}$ od, in one patient $(50 \%)$ symptoms resolved with dose reduction.

Conclusion We have previously published our long-term outcome data of IBD patients treated with thiopurines; $55 \%$ of these patients stopped thiopurine therapy due to therapeutic failure or side effects. The current data demonstrate that the majority of this refractory 